Review Article

\title{
The Curious Case of Cholangiocarcinoma: Opportunities for Environmental Health Scientists to Learn about a Complex Disease
}

\author{
William A. Suk (D), ${ }^{1}$ Vajarabhongsa Bhudhisawasdi, ${ }^{2}$ and Mathuros Ruchirawat ${ }^{3}$ \\ ${ }^{1}$ Superfund Research Program, National Institute of Environmental Health Sciences, Research Triangle Park, Durham, \\ NC 27709, USA \\ ${ }^{2}$ Surgery, Khon Kaen University, Changwat Khon Kaen, Khon Kaen 40002, Thailand \\ ${ }^{3}$ Research and Academic Affairs, Chulabhorn Research Institute, Bangkok 10210, Thailand
}

Correspondence should be addressed to William A. Suk; suk@niehs.nih.gov

Received 20 April 2018; Accepted 17 July 2018; Published 9 August 2018

Academic Editor: Felix A. Ogbo

Copyright ( 92018 William A. Suk et al. This is an open access article distributed under the Creative Commons Attribution License, which permits unrestricted use, distribution, and reproduction in any medium, provided the original work is properly cited.

Deaths from complex, noncommunicable diseases such as cancer are predicted to continue to increase worldwide, with low- and middle-income countries bearing the brunt of the burden. This problem requires a concentrated global effort to avoid devastating losses of life as well as economic losses. Cholangiocarcinoma (CCA) is a readily studied model of complex, noncommunicable disease, but it receives little attention outside of the scientific community in Southeast Asia. Here, we bring attention to the opportunity to study CCA as a model to understand the role of multiple, complex factors in cancer. These factors include allostatic load, individual genetic susceptibility, and environmental exposures such as chemicals, diet, socioeconomic factors, and psychosocial stress. The study of CCA offers a unique opportunity to make novel observations that could advance progress in prevention and intervention approaches for prevalent diseases that involve complex, multifactorial interactions.

\section{Introduction}

Deaths from cancer and other noncommunicable diseases are expected to continue to rise, especially in low- and middle-income countries (LMICs) [1,2]. The surge in cancer mortality is predicted to be especially dramatic in Southeast Asia, Southern China, and India, which face poverty, limited health care access, and other environmental conditions that intensify this burden [3]. This predicted burden on LMICs and the global increase in cancer, including in developed nations like the United States and the United Kingdom $[4,5]$, will cause catastrophic losses of life, in addition to economic devastation [3].

Complex diseases caused in part by environmental exposures are emerging as an increasing threat worldwide [1]. Our understanding of the rise in disease is complicated by the fact that human populations are incredibly diverse in their genes, lifestyles, daily environmental exposures, and other stressors, all of which can play a role in the multifactorial etiology of health and disease [6]. Disentangling the totality of exposures throughout the life-course and differences in the health impacts among individuals and populations is a challenge.

Studying diseases that are rare in the human population may offer a pathway to understanding many complex noncommunicable diseases. Because the affected populations are small and relatively well defined, it becomes easier to explore the environmental factors that may contribute to the disease. For example, researchers studying rare cancers have uncovered insights into diagnosing cancer, how cancer develops [7], gene mutations not previously implicated in cancer [8], and novel genes that can be used in diagnosis or classification of cancers [7]. Similarly, studying the interaction between aflatoxin and hepatitis B virus leading to liver cancer led to increased understanding of human environmental exposure levels, exposure assessment methods, metabolism of carcinogens and other toxicants, and mechanisms of carcinogenesis and other disease processes [9]. Thus, investigating rare 
diseases, including the underlying cellular mechanisms or genes involved, produces findings that can be translated to other more common diseases.

In this paper, we discuss some of the multiple, complex factors that may contribute to the rare bile duct cancer, cholangiocarcinoma (CCA), including diet and environmental exposures, genetic variations in susceptibility, coinfections and comorbidities, and socioeconomic and psychosocial factors. We illustrate how CCA is a real-world example of the effects of the exposome-the measure of all the endogenous and exogenous environmental exposures in an individual's lifetime and individual biological responses to these exposures [10]and argue that understanding the full picture of CCA promises to improve treatment and prevention of this disease, and help disentangle the many complex factors involved in other noncommunicable diseases.

\section{Cholangiocarcinoma as a Model Disease}

Though rare in most of the world, CCA incidence and mortality has increased in recent decades in many countries including LMICs, the United Kingdom, and United States [11-13]. However, it has received little attention and has not been well studied outside of the scientific community in Southeast Asia. To understand this increase in deaths and the burden that CCA presents, it is important to understand all the factors that contribute to the disease.

2.1. The Winding Pathway to CCA. In Southeast Asia, CCA is often found in people who consume raw or undercooked fish or sea plants that are infected with one of two different types of parasitic liver flukes. Indeed, both species of liver flukes that most commonly infect humans (Opisthorchis viverrini and Clonorchis sinensis) have been declared Group 1 carcinogens by the World Health Organization and the International Agency for Research on Cancer [14, 15]. Although CCA has been known to be a complex disease with multiple risk factors since 1942 [16], in much of the limited study to date, investigations have primarily explored the obvious risk factor of fluke infection [17].

A strong association between liver fluke infection and CCA has been observed, and a mechanism has been proposed [18-21]. The pathway by which liver fluke infection leads to CCA is complex with multiple junctions that can be influenced by environmental exposures. It is hypothesized that the fluke itself resides in the bile ducts where it injures the lining and produces abnormalities such as advanced periductal fibrosis. This in turn activates the body's woundrepair response, which can lead to altered cell states and DNA damage [20].

In addition, substances that damage DNA may be produced by the body's inflammatory response to the infection [20-22]. Yet another source of cell modification and cancer risk includes substances directly secreted by the fluke. This storm of cellular modifications and DNA damage may lead to fixed genetic alterations that may contribute to cancer [18-21].

Thus, combined with other environmental factors, the liver flukes themselves as well as the processes they set into motion have ample time to cause the DNA damage that can ultimately lead to cancer [18-22]. Importantly, while liver fluke infection is strongly associated with CCA, it is not required for the disease to occur. Many cases have been documented in the absence of fluke infection [23], and in animal and human studies, liver fluke infection leads to CCA in only a subset of those infected $[20,24]$.

Whether or not an infection progresses to CCA is determined by the duration of the infection, intensity of the infection, genetics of the host, genetics of the liver flukes themselves, other viral infections, other diseases, diet, and other environmental exposures (Figure 1) [20, 25, 26]. Indeed, CCA has possibly the highest number of causative risk factors among all human malignancies [27] in addition to complex relationships between factors [26].

2.2. Diet and Other Environmental Exposures. In addition to the common practice of eating traditional dishes using raw or fermented fish that may carry liver flukes, a diet high in nitrosamines from salted fish fermented meats, sausages, and betel nuts is thought to contribute to the high incidence of CCA [21, 26, 28]. Animal studies suggest that nitrosamines not only contribute to cancer formation but in some cases may be required for carcinogenesis. For example, in hamsters, bile-duct wounding plus dietary nitrosamines resulted in development of cancerous lesions, while neither state alone resulted in lesions [29]. Similar findings were reported in other studies where hamsters infected with liver flukes for less than seven months developed cancerous lesions only when subcarcinogenic doses of nitrosamines were added to the diet [30].

Heavy cigarette smoking and alcohol consumption are other sources of nitrosamines and have been suspected to increase risk of CCA $[12,31]$. The International Agency for Research on Cancer has also classified aflatoxins, which people can be exposed to through their diet, and arsenic, which they could be exposed to through drinking water, as carcinogens of the liver and bile duct with sufficient evidence and limited evidence, respectively [32].

Occupational exposures, including plutonium, aflatoxin, and vinyl chloride, have also been associated with liver cancer and CCA [32]. In Japan, occupational exposure to solvents has been associated with CCA [33]. Similarly, increased risk of CCA has been reported in workers exposed to asbestos, which researchers hypothesize involves an inflammatory pathway similar to liver fluke infection [34]. In Thailand, there has been some investigation into the relationship between occupational exposure to agricultural chemicals and CCA [35], but more information is needed about the potential role of these compounds, particularly in combination with other risk factors like liver fluke infection [26]. Other exposures that possibly have a relationship with CCA but require further study include polychlorinated biphenyls, dioxins, arsenic, and trichloroethylene [32, 36].

2.3. Genes and Genetic Variations. As with many cancers, individual differences play a role in the risk for CCA. Among CCA cases, only a subset has the long and intense 


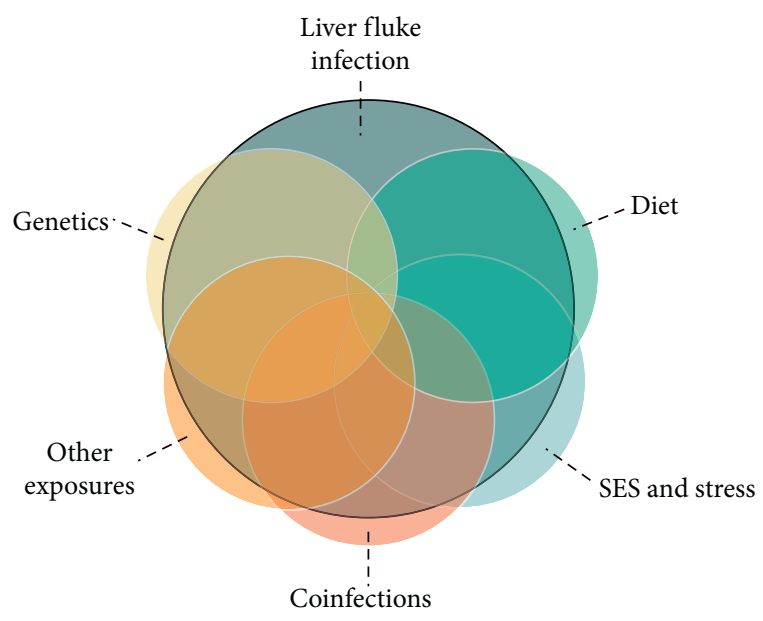

Figure 1: The complex factors involved in the pathway to CCA. Other environmental exposures may include nitrosamines, polycyclic aromatic hydrocarbons, arsenic, aflatoxin, and occupational exposures.

inflammatory response to the flukes that contributes to cancer. For example, family history of cancer has been found to be related to CCA incidence [26]. It is further hypothesized that these people have a modified or abnormal regulation of inflammatory cytokine production in response to fluke infection that increases their risk of developing bile duct cancer, and that differences in inflammatory responses could be due to genetic variations [20]. At least one researcher has suggested that dietary and other exposures lead to CCA only in the presence of polymorphisms in DNA repair genes [37]; others are exploring the connection with variation in genes controlling metabolic functions, growth factors, and metabolism of xenobiotics [26]. Exploring these questions will lead to important discoveries. For example, in an early study, whole exome sequencing of CCA cases associated with $O$. viverrini infection identified mutations in 10 genes that were not previously implicated in cancer [8].

In addition, genetic variation among infectious vectors affects the course of complex diseases. For example, analysis of the genome and trancriptomes of $O$. viverrini and $C$. sinensis has found major differences between the two similar flukes, including differences in genes that alter proteins that play a large role in tissue migration, immune system evasion, and feeding-all stages that are crucial to the parasite's survival in the human host [19]. These genes and proteins represent possible targets for drugs or vaccines and need further study. Studying CCA will yield greater understanding of this process. The availability of the parasite's genome offers an opportunity for researchers to explore differences in infection rates among populations and the underlying causes, which can also inform comparative studies of understudied diseases $[19,38]$.

2.4. Coinfections and Comorbidities. Coinfections contribute to disease etiology and can exacerbate severity [39]. In the case of CCA, it is suggested that carcinogenic liver flukes have a symbiotic relationship with bacterium of the helicobacter species [39-44]. Infection with certain helicobacter species, Helicobacter billis and Helicobacter hepaticus, are thought to be involved in CCA based on studies in animal models [39] and in humans [43]. But results of efforts to detect these species in humans with bile duct cancer have been variable, though differences in methodology should be noted $[39,43]$. To more fully understand the role of coinfections in complex diseases such as CCA, standardized diagnostic techniques such as PCR assays, immunohistochemistry antigens, and serological methods should be developed to promote early diagnosis and improve outcomes for patients [39]. Other coinfections whose relationships with CCA have been suggested but require further study include hepatitis $\mathrm{C}$ and hepatitis B [12,45-47], Epstein-Barr [48], and roundworms (Ascaris lumbricoides) [12].

Comorbidities are known to increase disease mortality and complicate intervention approaches [49]. In the case of CCA, some scientists have described the disease as a complication of chronic inflammation of the biliary system, or primary sclerosing cholangitis [14]. Indeed, an increased risk for CCA has been found among people who have a surgical biliary drainage procedure called anastomosis. Inflammation caused by the procedure is thought to be the inciting factor $[12,50]$. Other comorbidities that may be related to CCA but require further study include inflammatory bowel disease, obesity, nonalcoholic liver disease, diabetes, and cirrhosis [17, 47, 48, 51, 52]. Studying comorbidities, such as those associated with CCA, can help researchers understand the influence of external exposures and other factors as well as shared underlying genes and pathways [49].

2.5. Social, Economic, and Psychosocial Factors. In Southeast Asia, more than 40 million people are infected with liver flukes. While liver flukes can be killed with praziquantel, people in Southeast Asia often get reinfected because of the persistent practice of eating raw fish, and due to socioeconomic factors that can delay or prevent access to treatment. These conditions make it common for people in this region to remain infected for long periods of time or even their whole lives, or to experience multiple cycles of infection followed by treatment $[14,20,53]$. A disproportionate number of CCA deaths in Thailand also occur in men, who often represent the major income earners for families, compounding the economic burden [20].

In addition, people in many rural communities in Southeast Asia are affected by a transition from farming to a more industrial economy, which may bring economic disparities and psychosocial stress [54-56]. The role of these factors in disease onset and progression has become an important area of research in recent years, with more attention focusing on the urgent need to understand how social and psychological factors are translated into disease risk [57-59]. For example, poverty exerts a chronic psychological stress on individuals and populations that has important implications for disease risk both by contributing to allostatic load and by predisposing populations to alcohol abuse and other risky behaviors [60]. 
The culturally important practice of food sharing, which promotes social cohesion and connection, has also been found to play a role in the risk for liver fluke infection, where households with more food sharing connections were more likely to have the infection [28]. These practices and other factors in human social ecology have been suggested as important considerations in understanding the transmission dynamics of liver fluke infection and other similar illnesses [28].

Other social and economic factors may be protective against CCA. For example, education level was found to have an inverse relationship with risk of CCA [26]. Other factors that did not have significant relationships, such as marital status and occupation, have been suggested to represent a range of factors that may have complex relationships with other CCA factors without being directly related to disease outcome [26].

Exploring these factors and how they contribute to or mitigate the risk of disease in the context of CCA may yield many important insights of relevance to other diseases, such as cardiovascular disease, immune suppression, respiratory diseases, and metabolic conditions [57, 58], where populations face economic or social stressors.

2.6. Considerations for Prevention and Intervention. Our current policies and infrastructure encourage data collection on few environmental exposures and neglect the unique needs of many regions and countries in the developing world for prevention and intervention strategies. These policies result in many missed opportunities for intervention along the pathway to noncommunicable disease. Understanding how to design policy and effective interventions for such complex conditions is crucial for stemming the tide of CCA and other noncommunicable diseases.

Like other multifactorial diseases that require a comprehensive approach for effective prevention [37], regional prevention for CCA will require interventions targeted at infections or exposures at both the national and local level. For example, there is evidence that liver flukes adapt and coevolve with their animal and possibly human hosts in their local region $[25,26]$. In addition, it is possible that humans in certain regions may develop disease tolerance, as has been seen with other helminth infections [61-63]. Therefore, mass administration of praziquantel may not be advisable, especially since repeated cycles of treatment followed by reinfection is associated with development of cancer [26] and may increase the risk of flukes in a region becoming drug resistant [25].

An alternative is targeted treatment based on infection intensity, genetic background, culture, and diet $[26,28,60,64]$. Understanding the importance of local perceptions, social networks, and other factors will inform interventions that target key disease risk factors while promoting important local customs and improving intervention outcomes $[28,64]$. Moving towards a more holistic approach that integrates environmental contributions, evolution, biology, psychology, and social or behavioral dimensions will provide valuable insight towards addressing a variety of complex public health problems in addition to CCA $[26,60]$.
Early detection is another important part of prevention and intervention. In complex diseases that involve interplay between infection, inflammation, and environment, it is imperative to embrace advances in gene sequencing and expression to accelerate biomarker discovery to identify the early-stage biological processes that lead to cancer or other complex diseases. With CCA, a noninvasive, specific, and sensitive diagnostic marker is needed to increase early detection and treatment to improve survival rates $[11,65]$. The stages between liver fluke infection and cancer are well characterized, but tests are needed to determine when an individual is in the midst of one of these stages so that intervention can begin. For instance, elevated concentrations of IL-6 in plasma have been shown to be a marker for the advanced fibrotic changes in the liver ducts that accompany bile duct cancer [20]. The Thai Ministry of Public Health has begun using plasma IL-6 screening, along with other tests, to detect early liver cancer in areas where infection with the $O$. viverrini liver fluke are common [20]. Advances in gene sequencing are revealing more about the biological activity involved in CCA and could possibly be used to diagnose infections with specific flukes [66]. Researchers also point to the need to make use of proteomics, or the large-scale study of proteins and their functions, to rapidly compare multiple pathways of protein expression in infected people who progress to cancer and those who do not [20].

Cancer and other complex, noncommunicable diseases have heterogeneous causes and mechanisms as well as highly variable geographic distribution. While efforts to prevent disease and control cancer must be individualized by location [67], exploring the many complex factors that contribute to CCA risk may help identify parallels that can be translated to other diseases and different populations.

\section{Conclusions}

Largely overlooked outside of the scientific community in Southeast Asia, CCA is a readily studied model that could improve our understanding of cancer [37] and potentially a large swath of other noncommunicable diseases. Many of the steps along the pathway from liver fluke infection to cancer are known, and a well-defined animal model of the disease is in place [20].

Many different cancers result from a scenario similar to CCA - an interaction between infectious agents, environmental exposures, and genetic susceptibility that increases allostatic load and can lead to disease [68]. While we currently lack many of the tools needed to get a full picture of the health impacts of complex environmental exposures [69], working to understand complex rare diseases like CCA provides an opportunity to develop such tools and improve our understanding of the interplay of multiple, heterogeneous factors in disease. It is time to focus more research attention on this long-neglected disease.

\section{Conflicts of Interest}

The authors declare that they have no conflicts of interest. 


\section{Authors' Contributions}

Vajarabhongsa Bhudhisawasdi and Mathuros Ruchirawat contributed equally to this work.

\section{Acknowledgments}

The authors wish to acknowledge science writers Adeline Lopez and Angela Spivey, contractors with MDB, Inc., for the Superfund Research Program, for writing and editorial assistance. They also wish to acknowledge Brittany Trottier, a health specialist with the Superfund Research Program, for reviewing the manuscript and providing logistical support.

\section{References}

[1] S. S. Lim, T. Vos, A. D. Flaxman et al., "A comparative risk assessment of burden of disease and injury attributable to 67 risk factors and risk factor clusters in 21 regions, 1990-2010: a systematic analysis for the Global Burden of Disease Study 2010," The Lancet, vol. 380, no. 9859, pp. 2224-2260, 2012.

[2] GBD 2016 Causes of Death Collaborators, "Global, regional, and national age-sex specific mortality for 264 causes of death, 1980-2016: a systematic analysis for the Global Burden of Disease Study 2016," The Lancet, vol. 390, no. 10100, pp. 1151-1210, 2017.

[3] F. M. G. J. Knaul, R. Atun, and A. Bhadelia, Closing the Cancer Divide: An Equity Imperative: Global Equity Initiative, Harvard University, Cambridge, MA, USA, 2012.

[4] A. B. Ryerson, C. R. Eheman, S. F. Altekruse et al., "Annual report to the nation on the status of cancer, 1975-2012, featuring the increasing incidence of liver cancer," Cancer, vol. 122, no. 9, pp. 1312-1337, 2016.

[5] GBD 2016 Risk Factors Collaborators, "Global, regional, and national comparative risk assessment of 84 behavioural, environmental and occupational, and metabolic risks or clusters of risks, 1990-2016: a systematic analysis for the Global Burden of Disease Study 2016," The Lancet, vol. 390, no. 10100, pp. 1345-1422, 2017.

[6] J. Stingone, G. Buck-Louis, S. Nakayama et al., "Toward greater implementation of the exposome research paradigm within environmental epidemiology," Annual Review of Public Health, vol. 38, pp. 315-327, 2017.

[7] F. Jamshidi, T. O. Nielsen, and D. G. Huntsman, "Cancer genomics: why rare is valuable," Journal of Molecular Medicine, vol. 93, no. 4, pp. 369-381, 2015.

[8] C. K. Ong, C. Subimerb, C. Pairojkul et al., "Exome sequencing of liver fluke-associated cholangiocarcinoma," Nature Genetics, vol. 44, no. 6, pp. 690-693, 2012.

[9] C. P. Wild and Y. Y. Gong, "Mycotoxins and human disease: a largely ignored global health issue," Carcinogenesis, vol. 31, no. 1, pp. 71-82, 2010.

[10] C. P. Wild, "Complementing the genome with an "exposome": the outstanding challenge of environmental exposure measurement in molecular epidemiology," Cancer Epidemiology Biomarkers and Prevention, vol. 14, no. 8, pp. 1847-1850, 2005.

[11] G. K. Bonney, R. A. Craven, R. Prasad, A. F. Melcher, P. J. Selby, and R. E. Banks, "Circulating markers of biliary malignancy: opportunities in proteomics?, Lancet Oncology, vol. 9, no. 2, pp. 149-158, 2008.

[12] R. Al-Bahrani, Y. Abuetabh, N. Zeitouni, and C. Sergi, "Cholangiocarcinoma: risk factors, environmental influences and oncogenesis," Annals of Clinical \& Laboratory Science, vol. 43, no. 2, pp. 195-210, 2013.

[13] K. J. Yao, S. Jabbour, N. Parekh, Y. Lin, and R. A. Moss, "Increasing mortality in the United States from cholangiocarcinoma: an analysis of the National Center for Health Statistics Database," BMC Gastroenterology, vol. 16, no. 1, p. 117, 2016.

[14] IARC, "Infection with liver flukes (Opisthorchis viverrini, Opisthorchis felineus and Clonorchis sinensis)," IARC Monographs on the Evaluation of Carcinogenic Risks to Humans, vol. 61, pp. 121-175, 1994.

[15] IARC, "Opisthorchis viverrini and Clonorschis sinensis," IARC Monographs on the Evaluation of Carcinogenic Risks to Humans, vol. 100, pp. 341-370, 2012.

[16] W. Hueper, Occupational Tumors and Allied Diseases, Charles C Thomas, Springfield, IL, USA, 1942.

[17] H. R. Shin, J. K. Oh, E. Masuyer et al., "Epidemiology of cholangiocarcinoma: an update focusing on risk factors," Cancer Science, vol. 101, no. 3, pp. 579-585, 2010.

[18] J. M. Correia da Costa, N. Vale, M. J. Gouveia et al., "Schistosome and liver fluke derived catechol-estrogens and helminth associated cancers," Frontiers in Genetics, vol. 5, p. 444, 2014.

[19] N. D. Young, N. Nagarajan, S. J. Lin et al., "The Opisthorchis viverrini genome provides insights into life in the bile duct," Nature Communications, vol. 5, p. 4378, 2014.

[20] B. Sripa, P. J. Brindley, J. Mulvenna et al., "The tumorigenic liver fluke Opisthorchis viverrini-multiple pathways to cancer," Trends in Parasitology, vol. 28, no. 10, pp. 395-407, 2012.

[21] M. J. Smout, B. Sripa, T. Laha et al., "Infection with the carcinogenic human liver fluke, Opisthorchis viverrini," Molecular BioSystems, vol. 7, no. 5, pp. 1367-1375, 2011.

[22] P. Yongvanit, S. Pinlaor, and H. Bartsch, "Oxidative and nitrative DNA damage: key events in opisthorchiasis-induced carcinogenesis," Parasitology International, vol. 61, no. 1, pp. 130-135, 2012.

[23] W. Chan-On, M. L. Nairismagi, C. K. Ong et al., "Exome sequencing identifies distinct mutational patterns in liver fluke-related and non-infection-related bile duct cancers," Nature Genetics, vol. 45, no. 12, pp. 1474-1478, 2013.

[24] N. Songserm, J. Prasongwattana, P. Sithithaworn, B. Sripa, and V. Pipitkool, "Cholangiocarcinoma in experimental hamsters with long-standing Opisthorchis viverrini infection," Asian Pacific journal of cancer prevention, vol. 10, no. 2, pp. 299-302, 2009.

[25] P. Echaubard, B. Sripa, F. F. Mallory, and B. A. Wilcox, "The role of evolutionary biology in research and control of liver flukes in Southeast Asia. Infection, genetics and evolution," Journal of Molecular Epidemiology and Evolutionary Genetics in Infectious Diseases, vol. 43, pp. 381-397, 2016.

[26] J. A. Steele, C. H. Richter, P. Echaubard et al., "Thinking beyond Opisthorchis viverrini for risk of cholangiocarcinoma in the lower Mekong region: a systematic review and meta-analysis," Infectious Diseases of Poverty, vol. 7, no. 1, p. 44, 2018.

[27] G. Brandi, A. Farioli, A. Astolfi, G. Biasco, and S. Tavolari, "Genetic heterogeneity in cholangiocarcinoma: a major challenge for targeted therapies," Oncotarget, vol. 6, no. 17, pp. 14744-14753, 2015.

[28] P. Saenna, C. Hurst, P. Echaubard, B. A. Wilcox, and B. Sripa, "Fish sharing as a risk factor for Opisthorchis viverrini infection: evidence from two villages in north-eastern Thailand," Infectious Diseases of Poverty, vol. 6, no. 1, p. 66, 2017. 
[29] W. Thamavit, C. Pairojkul, D. Tiwawech, M. Itoh, T. Shirai, and N. Ito, "Promotion of cholangiocarcinogenesis in the hamster liver by bile duct ligation after dimethylnitrosamine initiation," Carcinogenesis, vol. 14, no. 11, pp. 2415-2417, 1993.

[30] C. Pairojkul, T. Shirai, S. Hirohashi et al., "Multistage carcinogenesis of liver-fluke-associated cholangiocarcinoma in Thailand," Princess Takamatsu Symposium, vol. 22, pp. 77-86, 1991.

[31] S. Honjo, P. Srivatanakul, H. Sriplung et al., "Genetic and environmental determinants of risk for cholangiocarcinoma via Opisthorchis viverrini in a densely infested area in Nakhon Phanom, northeast Thailand," International Journal of Cancer, vol. 117, no. 5, pp. 854-860, 2005.

[32] B. Charbotel, B. Fervers, and J. Droz, "Occupational exposures in rare cancers: a critical review of the literature," Critical Reviews in Oncology/Hematology, vol. 90, no. 2, pp. 99-134, 2014.

[33] M. M. Kirstein and A. Vogel, "Epidemiology and risk factors of cholangiocarcinoma," Visceral Medicine, vol. 32, no. 6, pp. 395-400, 2016.

[34] G. Brandi, S. Di Girolamo, A. Farioli et al., "Asbestos: a hidden player behind the cholangiocarcinoma increase? Findings from a case-control analysis," Cancer Causes Control, vol. 24, no. 5, pp. 911-918, 2013.

[35] K. Jeephet, S. Kamsa-Ard, V. Bhudhisawasdi, S. Kamsa-Ard, V. Luvira, and V. Luvira, "Association between pesticide use and cholangiocarcinoma," Asian Pacific Journal of Cancer Prevention, vol. 17, no. 8, pp. 3979-3982, 2016.

[36] V. Rapisarda, C. Loreto, M. Malaguarnera et al., "Hepatocellular carcinoma and the risk of occupational exposure," World Journal of Hepatology, vol. 8, no. 13, pp. 573-590, 2016.

[37] N. Songserm, S. Promthet, C. Pientong, T. Ekalaksananan, P. Chopjitt, and S. Wiangnon, "Gene-environment interaction involved in cholangiocarcinoma in the Thai population: polymorphisms of DNA repair genes, smoking and use of alcohol," BMJ Open, vol. 4, no. 10, article e005447, 2014.

[38] R. B. Gasser, P. Tan, B. T. Teh, S. Wongkham, and N. D. Young, "Genomics of worms, with an emphasis on Opisthorchis viverrini-opportunities for fundamental discovery and biomedical outcomes," Parasitology International, vol. 66, no. 4, pp. 341-345, 2016.

[39] F. K. Segura-Lopez, A. Guitron-Cantu, and J. Torres, "Association between Helicobacter spp. infections and hepatobiliary malignancies: a review," World Journal of Gastroenterology, vol. 21, no. 5, pp. 1414-1423, 2015.

[40] R. Deenonpoe, C. Chomvarin, C. Pairojkul et al., "The carcinogenic liver fluke Opisthorchis viverrini is a reservoir for species of Helicobacter. Asian Pacific journal of cancer prevention," Asian Pacific Journal of Cancer Prevention, vol. 16, no. 5, pp. 1751-1758, 2015.

[41] F. Aviles-Jimenez, A. Guitron, F. Segura-Lopez et al., "Microbiota studies in the bile duct strongly suggest a role for Helicobacter pylori in extrahepatic cholangiocarcinoma," Clinical Microbiology and Infection, vol. 22, no. 2, pp. 178. e11-178.e22, 2016.

[42] F. K. Segura-Lopez, F. Aviles-Jimenez, A. Guitron-Cantu et al., "Infection with Helicobacter bilis but not Helicobacter hepaticus was associated with extrahepatic cholangiocarcinoma," Helicobacter, vol. 20, no. 3, pp. 223-230, 2015.

[43] S. J. Kaewpitoon, R. A. Loyd, R. Rujirakul et al., "Helicobacter species are possible risk factors of cholangiocarcinoma,"
Asian Pacific Journal of Cancer Prevention, vol. 17, no. 1, pp. 37-44, 2016.

[44] P. Pisani, M. T. Whary, I. Nilsson et al., "Cross-reactivity between immune responses to Helicobacter bilis and Helicobacter pylori in a population in Thailand at high risk of developing cholangiocarcinoma," Clinical and Vaccine Immunology: CVI, vol. 15, no. 9, pp. 1363-1368, 2008.

[45] B. S. Lee, E. C. Park, S. W. Park, C. M. Nam, and J. Roh, "Hepatitis B virus infection, diabetes mellitus, and their synergism for cholangiocarcinoma development: a casecontrol study in Korea," World Journal of Gastroenterology, vol. 21, no. 2, pp. 502-510, 2015.

[46] P. Srivatanakul, S. Honjo, P. Kittiwatanachot, A. Jedpiyawongse, T. Khuhaprema, and M. Miwa, "Hepatitis viruses and risk of cholangiocarcinoma in northeast Thailand," Asian Pacific Journal of Cancer Prevention, vol. 11, no. 4, pp. 985-988, 2010.

[47] J. S. Chang, C. R. Tsai, and L. T. Chen, "Medical risk factors associated with cholangiocarcinoma in Taiwan: a populationbased case-control study," PLoS One, vol. 8, no. 7, Article ID e69981, 2013.

[48] IARC, Pathology and Genetics of Tumours of the Digestive System, World Health Organisation and International Agency for Research on Cancer, Lyon, France, 2000.

[49] J. Hu, C. Thomas, and S. Brunak, "Network biology concepts in complex disease comorbidities," Nature Reviews Genetics, vol. 17, pp. 615-629, 2016.

[50] A. Tocchi, G. Mazzoni, G. Liotta, L. Lepre, D. Cassini, and M. Miccini, "Late development of bile duct cancer in patients who had biliary-enteric drainage for benign disease: a followup study of more than 1,000 patients," Annals of Surgery, vol. 234, no. 2, pp. 210-214, 2001.

[51] S. Kahn, M. Toledano, and S. Taylor-Robinson, "Epidemiology, risk factors, and pathogenesis of cholangiocarcinoma," $H P B$, vol. 10, no. 2, pp. 77-82, 2008.

[52] H. Zhang, T. Yang, M. Wu, and F. Shen, "Intrahepatic cholangiocarcinoma: epidemiology, risk factors, diagnosis and surgical management," Cancer Letters, vol. 379, no. 2, pp. 198-205, 2016.

[53] P. Sithithaworn, R. H. Andrews, V. D. Nguyen et al., "The current status of opisthorchiasis and clonorchiasis in the Mekong Basin," Parasitology International, vol. 61, no. 1, pp. 10-16, 2012.

[54] J. Bircher and S. Kuruvilla, "Defining health by addressing individual, social, and environmental determinants: new opportunities for health care and public health," Journal of Public Health Policy, vol. 35, no. 3, pp. 363-386, 2014.

[55] J. Rigg, "Land, farming, livelihoods, and poverty: rethinking the links in the rural south," World Development, vol. 34, no. 1, pp. 180-202, 2006.

[56] M. Kelly, L. Strazdins, T. Dellora, S. Khamman, S. Seubsman, and A. Sleigh, "Thailand's work and health transition," International Labour Review, vol. 149, no. 3, pp. 373-386, 2010.

[57] T. Kamarck, "Psychosocial stress and cardiovascular disease: an exposure science perspective," Psychological Science Agenda, vol. 26, no. 4, 2012.

[58] M. R. Salleh, "Life event, stress and illness," Malaysian Journal of Medical Sciences, vol. 15, no. 4, pp. 9-18, 2008.

[59] S. C. Segerstrom and D. B. O'Connor, "Stress, health and illness: four challenges for the future," Psychology and Health, vol. 27, no. 2, pp. 128-140, 2012.

[60] B. A. Wilcox and P. Echaubard, "Balancing biomedical and ecological perspectives in research framing of liver fluke and 
cholangiocarcinoma in NE, Thailand," Parasitology International, vol. 66, no. 4, pp. 372-377, 2017.

[61] T. J. Little, D. M. Shuker, N. Colegrave, T. Day, and A. L. Graham, "The coevolution of virulence: tolerance in perspective," PLoS Pathogens, vol. 6, no. 9, article e1001006, 2010.

[62] J. E. Allen and T. A. Wynn, "Evolution of Th2 immunity: a rapid repair response to tissue destructive pathogens," PLoS Pathogens, vol. 7, no. 5, article e1002003, 2011.

[63] R. Medzhitov, D. S. Schneider, and M. P. Soares, "Disease tolerance as a defense strategy," Science, vol. 335, no. 6071, pp. 936-941, 2012.

[64] O. Zvonareva, P. Odermatt, E. A. Golovach et al., "Life by the river: neglected worm infection in western Siberia and pitfalls of a one-size-fits-all control approach," Critical Public Health, pp. 1-12, 2017.

[65] J. R. Skipworth, J. F. Timms, and S. P. Pereira, "Novel diagnostic and prognostic biomarkers in biliary tract cancer," Expert Opinion on Medical Diagnostics, vol. 7, no. 5, pp. 487-499, 2013.

[66] L. T. Phung, A. Loukas, P. J. Brindley, B. Sripa, and T. Laha, "Retrotransposon OV-RTE-1 from the carcinogenic liver fluke Opisthorchis viverrini: potential target for DNA-based diagnosis," Infection, Genetics and Evolution, vol. 21, pp. 443-451, 2014.

[67] C. P. Wild, "The role of cancer research in noncommunicable disease control," JNCI Journal of the National Cancer Institute, vol. 104, no. 14, pp. 1051-1058, 2012.

[68] B. S. McEwen and E. Stellar, "Stress and the individual. Mechanisms leading to disease," Archives of Internal Medicine, vol. 153, no. 18, pp. 2093-2101, 1993.

[69] M. M. Niedzwiecki and G. W. Miller, "The exposome paradigm in human health: lessons from the emory exposome summer course," Environmental Health Perspectives, vol. 125, no. 6, article 064502, 2017. 


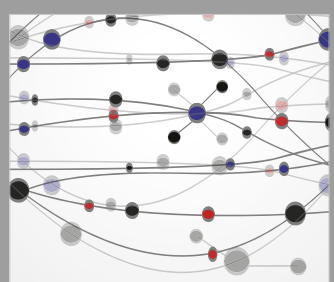

The Scientific World Journal
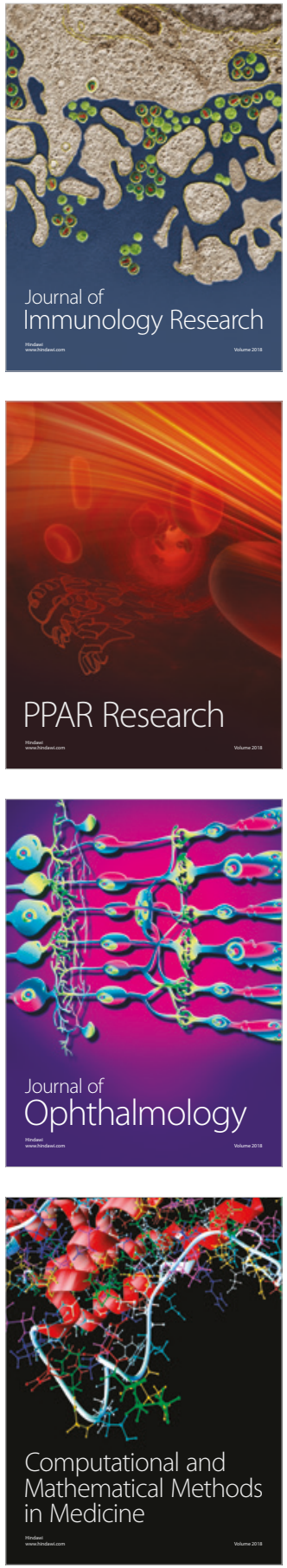

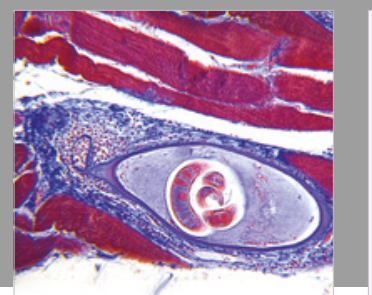

Gastroenterology Research and Practice

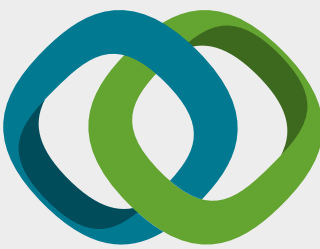

\section{Hindawi}

Submit your manuscripts at

www.hindawi.com
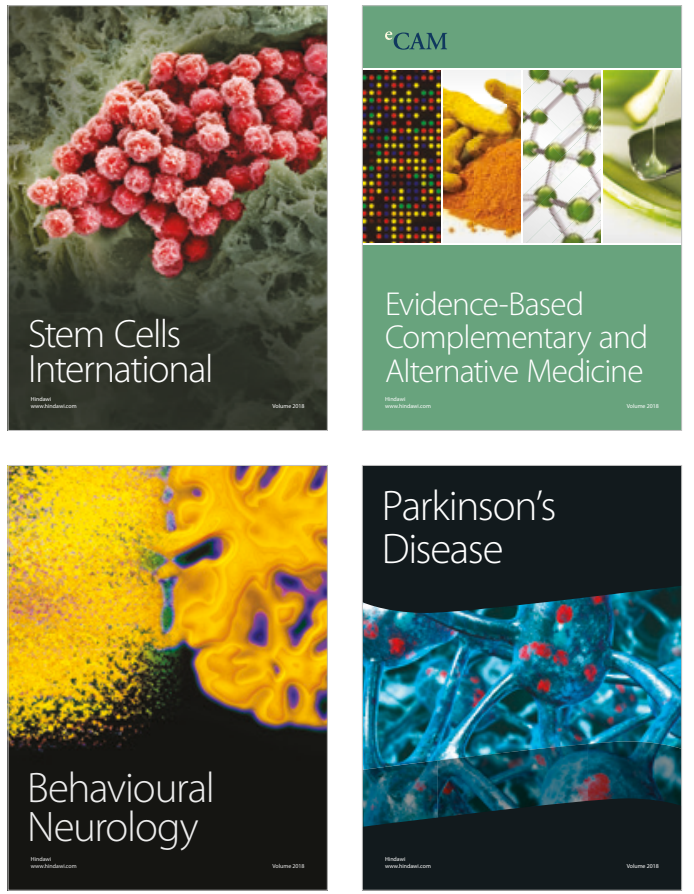

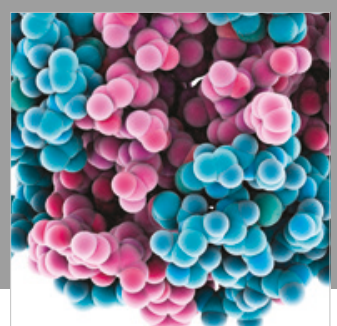

ournal of

Diabetes Research

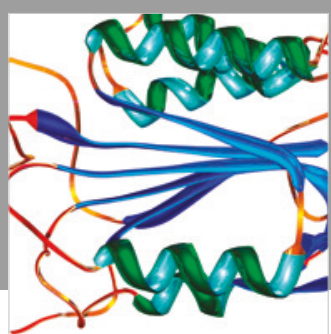

Disease Markers
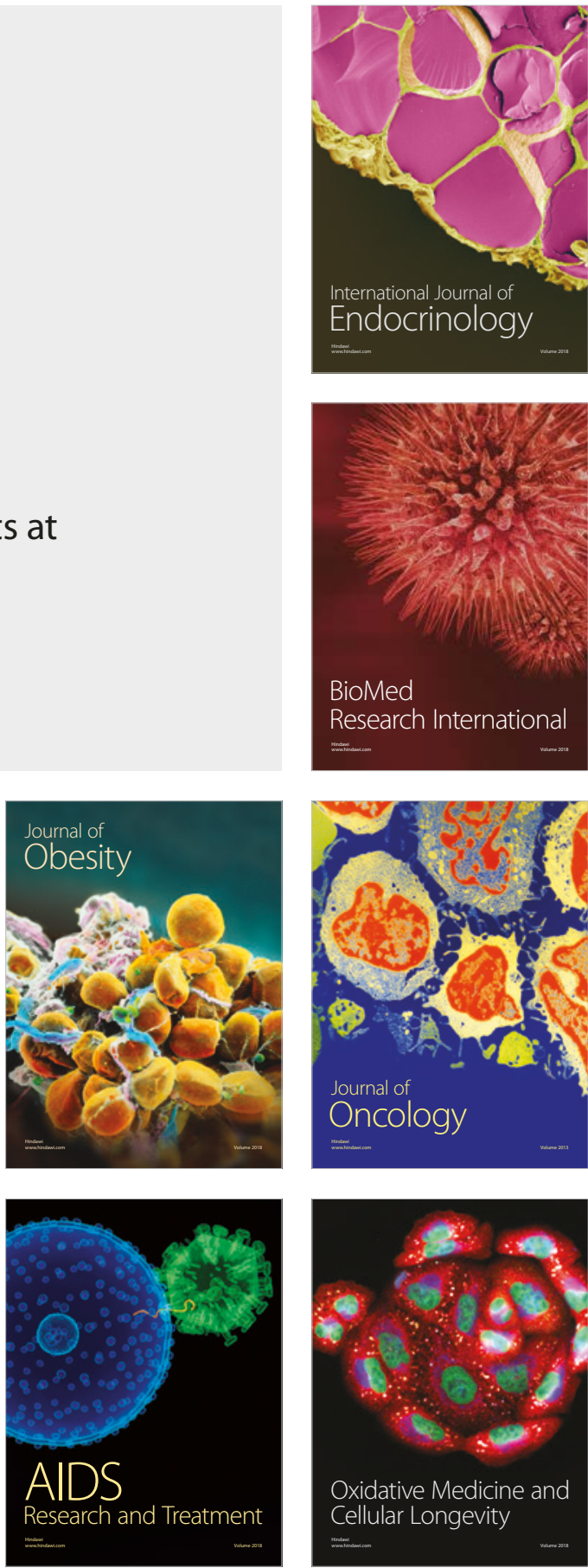